\title{
Genome Editing in Gene and Cell-Based Therapies
}

\section{Saranya Ganapathy and Sanjeev K Gupta*}

Advanced Biotech Lab, Ipca Laboratories Ltd., Kandivli, Mumbai, India

*Corresponding Author: Sanjeev K Gupta, Advanced Biotech Lab, Ipca Laboratories

Ltd., Kandivli, Mumbai, India.
Received: March 11, 2020

Published: April 14, 2020

(C) All rights are reserved by Saranya

Ganapathy and Sanjeev K Gupta.

\begin{abstract}
Site-specific genetic modification has become a realistic possibility after nearly 50 years of research in the field of gene editing/ therapy. With the need for introducing exogenous fragment DNA or removing/modifying endogenous gene in/from a specific site in the genome of interest, precise genetic tools are being carefully designed for implementation. Creating Double Stranded Breaks (DSBs) at targeted sites in the genome of interest followed by endogenous cell based repair (Homology Directed Repair (HDR) or Non-Homologous End Joining (NHEJ)) results in genomic modifications [1]. With this approach, it is possible to carry out gene insertion, augmentation, deletion, gene sequence modification and mutation correction, aiding in the development of better animal disease models, ex vivo and in vivo gene repair and engineered cell based therapies.

Keywords: Double Stranded Breaks (DSBs); Homology Directed Repair (HDR); Non-Homologous End Joining (NHEJ)
\end{abstract}

\section{Gene editing tools}

The main tools associated with gene editing are the viral vectors and nucleases. Use of retroviral vectors ( $\mathrm{Z}$-retroviral and later Lentiviral vectors) kick started the first-generation gene therapy for hematopoietic stem cells application. Also, uses of replicationdefective parvovirus (Adeno Associated Viral (AAV) vectors) started in the 1990s and continue to be explored for Hemophilia and liver disorders, by isolating variants and engineering novel capsids. Currently, AAV appears to be the most popular vector among the other viral and non-viral vectors and is being shown to be safe and effective in preclinical and clinical studies. Recent advances and modifications in the viral vectors to reduce the risk of genotoxicity/immunogenicity, and optimal dosing have led to substantial clinical progress - both for prophylactic and therapeutic purposes [2]. However, viral vectors could only be used for genome editing by gene addition.

Four versatile platform nucleases that have been widely used include Zinc finger nucleases (ZFN), Meganucleases, Transcription Activators Like Effector Nucleases (TALENs) and Clustered Regularly Interspaced Palindromic Repeats (CRISPR/Cas). These nucleases act by aiming at specific sites (using specific DNA binding proteins or guide RNA) in the target genome where DSBs are generated. Gene deletion/knockout is carried out by targeting coding regions resulting in indels/frameshift mutations/complete removal of the enhancer or coding region. Gene correction/modi- fication is carried out by creating targeted DSBs and introducing exogenous DNA fragment (donor DNA) through HDR. Donor DNA can be plasmid DNA, single stranded oligonucleotides or integrasedefective lentiviral/AAV vectors [3-6]. Gene regulation (Switching genes ON/OFF, modulation of expression levels, epigenetic regulation) has also become a possibility with engineered CRISPR/Cas $[7,8]$.

Factors that significantly impact the efficiency of genome/gene editing include: 1) Delivery of genome editing tools to the target cells (transfection/transduction efficiencies; dosage/expression levels of the tool components) $[9,10], 2)$ Specificity of nucleases (by creating improved versions of nucleases and other edit tool components) $[11,12]$ and sensitivity of detection of off-target effects (in silico models, by whole genome sequencing and ChIP based identification of binding/target sites) [13], 3) Tissue specific targeting in-vivo.

\section{Gene editing in gene/cell therapy}

Gene delivery in vivo involves targeting the genome editing components to specific organs/ tissues and target cell-specific expression. Recently, antisense oligonucleotide (ASO), Nusinersen, was approved by US-FDA and EMA. This therapy provides a promising treatment for neuromuscular disorder, spinal muscular dystrophy, which is caused by deletion or loss of function mutations of SMN1 gene. Other AAV based gene therapies have also been approved by 
US-FDA/EMA between 2014 -2018 for hemophilia and inherited retinal dystrophy.

On the other hand, ex vivo genome editing through cell engineering for cancer immunotherapies and blood disorders started with gene editing in Hematopoietic stem cells for allogeneic bone marrow transplantation. Later, autologous transplants of ex vivo modified cells brought in more advantages which helped to avoid immune complications. Engineered T cells are gradually becoming an efficient cancer therapy. To enhance T cell function, the cells are engineered with Chimeric Antigen Receptors (CAR) which can bind to specific proteins or carbohydrates and activates intracellular chimeric signaling domain that drives $\mathrm{T}$ cell activation. These CAR-T cells are generated by stable transfer of CAR genes to establish sustained receptor expression. Preclinical and clinical studies on CAR-T cells (using lentiviral, X-retroviral, transposons, CRISPRCas) have substantiated the theory. Some level of toxicity has been noted with this strategy due to off-tumor effects and cytokine release syndrome. Universal CAR-T cells ('off-the-shelf' drugs) are an attractive option which could be made possible with the help of multiplex CRISPR technology to remove endogenous T cell receptors, introduction of CAR in T cells, and/or knock out of immune check points [14].

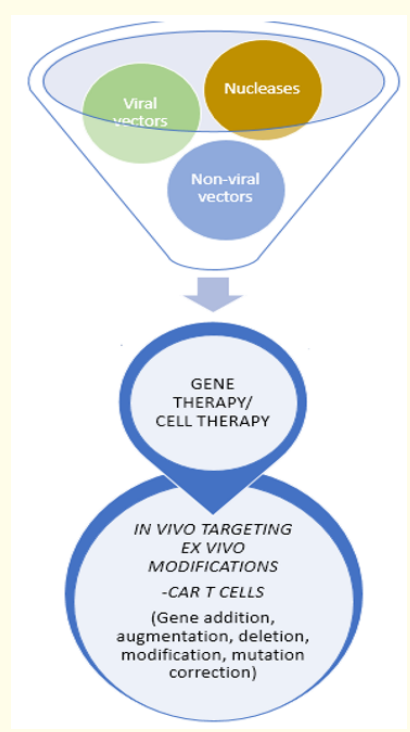

Figure 1: A snapshot of gene/cell based therapy.

Ability to modify genome sequences has paved the way to create treatment options for diverse inherited and acquired genetic/ epigenetic disorders in human, including major categories of diseases (hematological, neuromuscular, ocular, skin, respiratory disorders; cancer immunotherapies; anti-microbials). In contrast to protein based drugs, gene/cell based therapy might have a longer lasting effect owing to the permanent genomic changes it can offer. The germline modifications to avoid heritable diseases have now become a possibility owing to the advancements made in genome editing tools. However, rigorous safety and efficacy studies with somatic cells are warranted for further consideration and implementation of germline editing.

As of now, more than 2500 clinical studies have been initiated for a wide range of human disorders with more focus on cancer and monogenic diseases. So far, few gene therapies have been approved by US-FDA/ EMA for AAV-based therapy for lipoprotein lipase deficiency and retinal dystrophy; $\gamma$-retrovirus based therapy for treatment of Severe Combined Immunodeficiency (ADA-SCID); Autologous CAR-T cell therapies for non-hodgkins lymphoma and acute lymphoblastic leukemia $[15,16]$. Strimvellis, Luxturna, Kymriah, Yescarta, Kynamro, Nusinesren, to name a few, have all made it to the clinic with very promising results. Gene-editing products have moved to early phase of clinical trials, which include ZFN targeted CCR5 gene (HIV infection) and CRISPR/Cas targeted T-cells for programmed death-1 (PD1) knockout to treat esophageal cancer.

\section{Challenges and future aspects}

While the gene and cell therapy offers promise, the hefty price tags on these (ranging from $\$ 300,000$ to $\$ 850,000$ ) raise questions on sustainability and true cost-benefit analysis of these therapies. Broadly, insertional mutagenesis, genotoxicity, deleterious immune responses, and excessive $\mathrm{T}$ cell activation, depending on the mode of gene/cell therapy, are the bottle necks in the progress in this field. Efficiency and long term safety of clinical translation of genome engineering in specific targeted tissues remain a pressing challenge. However, potential for this field in positively impacting disease cure, therapies and personalized medicine have constantly aided in continued progress in overcoming these hurdles through development of strategies to mitigate the risks. The genetic disorders and life-threatening diseases, that were once beyond our reach and considered incurable, have now become treatable and gene/cell therapy is making that possible to the great extend

\section{Acknowledgement}

Authors would like to thank Ipca Laboratories Ltd., Mumbai, India for the extended support.

\section{Bibliography}

1. Takata M., et al. "Homologous recombination and non-homologous end-joining pathways of DNA double-strand break repair have overlapping roles in the maintenance of chromosomal integrity in vertebrate cells". EMBOJ 17 (1998): 5497-5508. 
2. Lundstrom K. "Viral Vectors in Gene Therapy". Diseases 6.2 (2018): 42 .

3. Renaud JB., et al. "Improved Genome Editing Efficiency and Flexibility Using Modified Oligonucleotides with TALEN and CRISPR-Cas9 Nucleases". Cell Reports 14 (2016): 2263-2272.

4. Chen F., et al. "High-frequency genome editing using ssDNA oligonucleotides with zinc-finger nucleases". Nature Methods 8 (2011): 753-755.

5. Gaj T., et al. "Targeted gene knock-in by homology-directed genome editing using Cas9 ribonucleoprotein and AAV donor delivery". Nucleic Acids Research 45.11 (2017): 98.

6. Devices CX., et al. "Engineered Viruses as Genome Editing Devices". Molecular Therapy 24.3 (2016): 447-457.

7. Gilbert LA., et al. "CRISPR-mediated modular RNA-guided regulation of transcription in eukaryotes". Cell 154 (2013): 442-451.

8. Qi LS., et al. "Repurposing CRISPR as an RNA-guided platform for sequence-specific control of gene expression". Cell 152 (2013): 1173-1183.

9. Wang M., et al. "Non-viral delivery of genome-editing nucleases for gene therapy". Gene Therapy 24 (2017): 144-150.

10. Yin H., et al. "Delivery technologies for genome editing". Nature Reviews Drug Discovery 16 (2017): 387-399.

11. Miller JC., et al. "Enhancing gene editing specificity by attenuating DNA cleavage kinetics". Nature Biotechnology 37 (2019): 945-952.

12. Liu RM., et al. "Synthetic chimeric nucleases function for efficient genome editing". Nature Communications 10 (2019): 5524.

13. Li J., et al. "Advances in detecting and reducing off-target effects generated by CRISPR-mediated genome editing". Journal of Genetics and Genomics 46.11 (2019): 513-521.

14. Puig-Saus C., et al. "Gene editing: Towards the third generation of adoptive T-cell transfer therapies". Immuno-Oncology Technology 1 (2019): 19-26.

15. Golchin A., et al. "Biological Products: Cellular Therapy and FDA Approved Products". Stem Cell Rev Stem Cell Reviews and Reports 15 (2019): 166-175.
16. Anguela XM., et al. "Entering the Modern Era of Gene Therapy". High Annual Review of Medicine 70.1 (2019): 273-288.

\section{Assets from publication with us}

- Prompt Acknowledgement after receiving the article

- Thorough Double blinded peer review

- Rapid Publication

- Issue of Publication Certificate

- High visibility of your Published work

Website: https://www.actascientific.com/

Submit Article: https://www.actascientific.com/submission.php Email us: editor@actascientific.com

Contact us: +919182824667 